\title{
INDOOR LOCALIZATION OF RFID-EQUIPPED MOVABLE ASSETS USING MOBILE READER BASED ON REFERENCE TAGS CLUSTERING
}

\author{
A. Motamedi \\ Individualized Program (INDI), Concordia University, \\ 2145 Mackay Street, S204, Montreal, Quebec, Canada H3G 2J2
}

M. M. Soltani

Building, Civil and Environmental Engineering, Concordia University, 1455 de Maisonneuve Blvd. West, EV-6.139, Montreal, Quebec, Canada H3G 1 M8

* A. Hammad

Concordia Institute for Information Systems Engineering, Concordia University, 1515 Ste-Catherine Street West, EV7.643, Montreal, Quebec, Canada H3G 2W1

(*Corresponding author: hammad@ciise.concordia.ca) 


\begin{abstract}
Indoor localization has gained importance as it has the potential to improve various processes related to the lifecycle management of facilities and to deliver personalized and location-based services. Radio Frequency Identification (RFID) based systems, on the other hand, have been widely used in different applications in construction and maintenance. This paper investigates the usage of RFID technology for indoor localization of RFID equipped movable assets during the operation phase of facilities. The location-related data on RFID tags attached to fixed assets are extracted from a Building Information Model (BIM) and can provide context-aware information inside the building which can improve Facilities Management (FM) processes. The paper proposes a new approach to use received signals from available reference tags in the building attached to fixed assets to locate movable assets. The approach uses signal pattern matching and clustering algorithms for localization. As a result, a user equipped with an RFID reader is able to estimate the location of target assets, without having access to any RealTime Location System (RTLS) infrastructure. A case study is performed to demonstrate the feasibility of proposed methods.
\end{abstract}

\title{
KEYWORDS
}

Asset localization, RFID, Location-based services, Pattern matching, Clustering

\section{INTRODUCTION}

The localization problem has received considerable attention in the areas of pervasive computing as many applications need to know where objects are located. Location information can be used by occupants unfamiliar with a building to navigate and find their destinations. Additionally, facilities management (FM) personnel can be provided with locations of assets in order to decrease their search time for assets. Hence, indoor location information is especially valuable as it has the potential to improve the utilization and maintenance of facilities. Furthermore, location information is central to personalized applications in different areas and it is the basis for the delivery of personalized and location-based services (LBS). It is the basis for context awareness within the building, which involves an automatic recognition of the user's location and activity (Zhao et al., 2007; Papapostolou and Chaouchi, 2011).

Radio Frequency Identification (RFID) is a type of automatic identification technology in which radio frequencies are used to capture and transmit data (Aimglobal, 2008). It has been employed for localization in indoor environments in various research projects. In RFID-based localization systems, tags or readers can be the targets for localization. In tag localization, the RFID tag is attached to the target component for localization. In most of the settings, in order to track the target tag, readers or reference tags with known locations are deployed as reference points and a positioning technique is applied for estimating the location of the tag. In reader localization, usually reference tags are deployed as reference points for localization of the reader. RF-based localization methods can be categorized into five major groups: (1) Lateration using techniques such as: Time of Arrival (ToA), Time Difference of Arrival (TDoA), Phase of Arrival (POA), signal attenuation, and hop-based; (2) Angulation; (3) Fingerprinting (scene analysis); (4) Proximity; and (5) Neighbourhood. LANDMARC (Ni et al., 2003), has been a foundation for 
many neighbourhood-based solutions. It uses active tags that are attached to target assets and fixed reference tags with known locations that are placed in the sensing area. Sanpechuda and Kovavisaruch (2008), Papapostolou and Chaouchi (2011), and Li and Becerik-Gerber (2011) provided thorough surveys and comparisons among various projects for tag and reader localization.

The framework developed in our previous research has proposed adding structured information taken from the BIM database to RFID tags attached to building assets (Motamedi and Hammad 2009). The stored information on tags is beneficial for several lifecycle processes and is used by various stakeholders. In this framework, every asset is a potential target for tagging. Having tags attached to assets results in a massive tag cloud in the building. The current paper builds on the above-mentioned framework for achieving the following objectives: (1) to investigate new methods for localizing RFID-equipped movable assets during the operation and maintenance phase without having access to a wired Real Time Location System (RTLS) infrastructure, and (2) to investigate the applicability of the proposed methods using case studies.

\section{PROPOSED METHOD}

In our approach, the user who is searching for assets is equipped with a handheld RFID reader and is able to read the content of the tags from a distance to locate fixed and movable assets. The approach is based on the assumption that relatively long-range RFID tags are attached to assets. RFID tags that are attached to fixed assets contain their exact location coordinates and are available throughout the building. Moreover, it is assumed that the target tags are stationary for the period of localization and the user equipped with a handheld reader is moving within the facility to collect Received Signal Strength Indicator (RSSI) values in order to locate assets. The reference tags that exhibit similar signal patterns to those of the target tag are identified. A group of tags that show similar signal patterns are considered to be spatially adjacent. This similarity of patterns stems from the fact that the radio signals are affected by similar environmental effects for neighbouring tags. Consequently, this method does not use RSSI values to estimate the distance between the reader and tags due to the unreliability of this conversion in indoor environments. Finally, a subgroup of reference tags is selected and their coordinates are used for localizing the target tag. The proposed method is called Cluster-based Movable Tag Localization (CMTL) throughout the paper.

The Collected RSSI values received from reference tags attached to fixed assets and from the tag attached to the target asset are processed by the data processing module which includes: filtering logged RSSI values to eliminate the values that are out of range as the result of sudden noises, errors in recording data, hardware errors, etc.; data averaging and pattern matching to compare the pattern of the RSSI of the target tag with all reference tags using a pattern matching algorithm; and clustering to group reference tags considering the result of pattern matching and their spatial distribution. The location of the target asset is estimated based on the result of the pattern matching, clustering and other information such as spatial constraints. After estimating the location of the target tag, it is shown on the floor plan. The method can also be used to locate a group of movable assets by collecting RSSI values for all target tags while moving in the facility. The data are then processed to calculate the locations of all target tags.

\section{Pattern Matching Algorithm}

During the data collection, it is assumed that there are $n$ reference tags and $p$ target tags in the area. $R_{i}[i \in(1, n)]$ denotes the $i^{\text {th }}$ reference tag. $T_{j}[j \in(1, p)]$ denotes the $j^{\text {th }}$ target tag. Data 
collection happens at $m$ different data collection steps. $R S S_{S}^{R_{i}}$ denotes the averaged RSSI value for the $i^{\text {th }}$ reference tag at the $s^{\text {th }}$ data collection step after filtering. The goal of pattern matching is to determine which reference tags $\left(R_{i}\right)$ show similar signal patterns to the signal pattern received from the target tag $\left(T_{j}\right)$. The least square difference method is employed to calculate the similarity of reference tags to the target tag. $\beta_{R_{j}}^{T_{i}}$ is the distance indicator (pattern dissimilarity) value between the $i^{\text {th }}$ reference tag and the $j^{\text {th }}$ target tag after $m$ data collection steps (Equation 1 ). The matrix of $\beta$ (Equation 2) is constructed using the calculated values from Equation 1. The $\beta$ values in the $j^{\text {th }}$ column of the matrix indicate the distance indicators for each reference tag to the $j^{\text {th }}$ target tag. The least $\beta$ value in each column shows the reference tag that is assumably closer to the associated target tag.

\section{Identifying the Target Area by Clustering Reference Tags}

There are cases that some reference tags which are not spatially close to the target tag show similar signal patterns to that target tag. This can happen randomly or can be caused by the movement pattern of the user while collecting data and the layout of the building due to the symmetry of the distribution of reference tags with respect to the data collection path. For example if the user walks in a corridor where the rooms are located on two sides, there might be cases that reference tags located in different rooms across the corridor show similar signal patterns due to symmetry. Figure 1(a) shows an example layout of several reference tags and a target tag. Figure 1(b) shows the similarity of each reference tag represented by a circle where the diameter of the circles is inversely proportional to the $\beta$ value. LANDMARC method selects the best $k$ reference tags based on the $\beta$ values sorting and uses weighted averaging to locate the target tag. However, this technique may select reference tags that are far from the target. Therefore, the localization based on LANDMARC method suffers from a large error as shown in Figure 1(c).

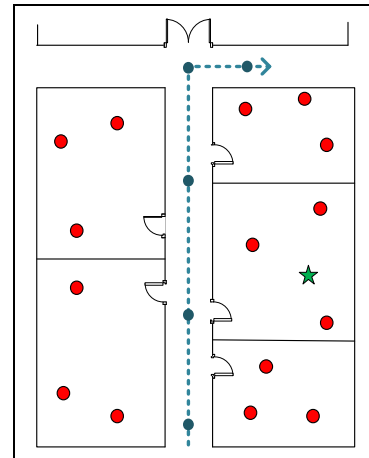

(a) Location of reference and target tags

$\star$ Target tag

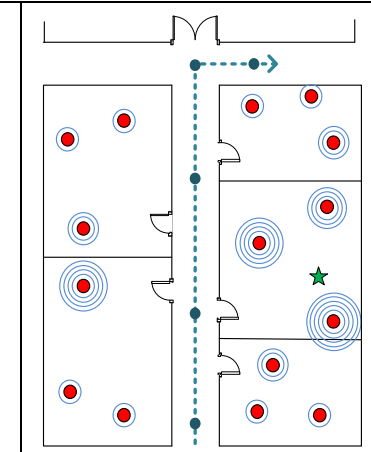

(b) Similarity of reference tags

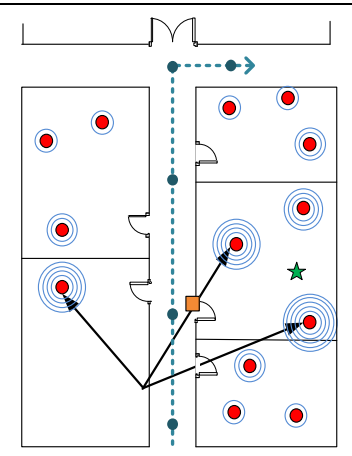

(c) Best three reference tags based on similarity

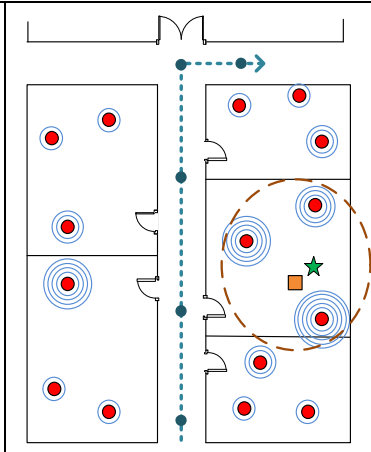

(d) Best three reference tags based on clustering $\begin{array}{llll}\begin{array}{l}\text { Reference } \\ \text { tag }\end{array} & \begin{array}{l}\text { Estimated location } \\ \text { of target tag }\end{array} & \begin{array}{l}\text { Signal } \\ \text { similarity }\end{array} & \begin{array}{l}\text { Data collection point } \\ \text { and path }\end{array}\end{array}$

Figure 1 - Reference tags clustering

The solution to this problem is to form clusters of reference tags that are spatially close. The target localization can be performed within the selected cluster, as shown in the Figure 1(d). However, clustering of reference tags based only on spatial closeness (nearness) of the tags does not necessarily lead to the best results. For example, Figure 2(a) shows a case where spatial clustering will not lead to the optimum selection of reference tags for localization. However, 
clustering of reference tags based only on spatial closeness (nearness) of the tags does not necessarily lead to the best results. The proposed method for clustering uses combination of two criteria for selecting members of each cluster: (1) closeness of reference tags: by selecting the reference tags that are spatially close to each other using algorithms such as k-means (Kanungo et al., 2002), and (2) similarity of reference tags to the target: by selecting tags that have similar signal pattern to that of the target tag using $\beta$ values. Consequently, by using the CMTL method, target tags that show similar signal pattern to the one of the target and at the same time are in close proximity of each other are chosen as the target cluster. Figure 2(b) shows how this clustering method chooses a group of tags that is spatially close and at the same time shows high signal pattern similarity. The steps to form clusters and chose the target cluster are as follows:

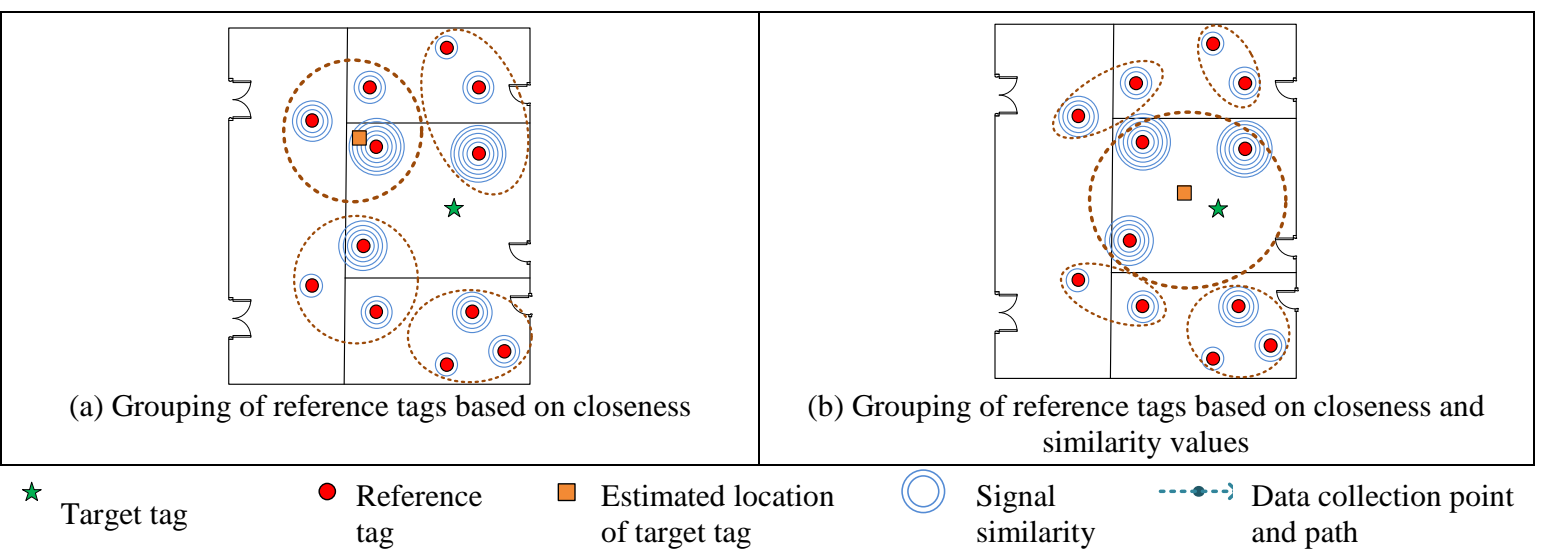

Figure 2 - Multi criteria clustering vs. single criterion

Calculating spatial closeness of clusters members: It is assumed that there are $z$ clusters available in the environment. $\mathrm{G}_{\mathrm{r}}[r \in(1, z)]$ denotes the $r^{\text {th }}$ cluster and $o_{G_{r}}$ denotes the total number of reference tags in the cluster. First, the $\mathrm{x}$ and $\mathrm{y}$ coordinates of the centroid point for each group are calculated. $x_{G_{r}}^{C}$ and $y_{G_{r}}^{C}$ denote the coordinates for the centroid point of $r^{\text {th }}$ group. $x_{R_{e} G_{r}}$ and $y_{R_{e}^{G_{r}}}$ denote the coordinates of the $\mathrm{e}^{\text {th }}$ member of the group. The total of the distances of each group member to the centroid of the group is calculated using Equation 3 and normalized using Equation 4.

Calculating the signal pattern similarity of members: In this stage the average signal pattern dissimilarity $(\beta)$ of each member of the group $\left(R_{e}^{G_{r}}\right)$ to the target tag $\left(T_{u}\right)$ is calculated using Equation 5 and the value is normalized using Equation 6.

Selecting the target cluster: The target cluster is selected based on two values calculated using Equations 4 and 6. $K_{G_{r}}$ denotes the score of each multi-dimensional cluster based on two criteria as shown in Equation 7. The weights, $w_{D}$ and $w_{\beta}$, can be adjusted based on the layout of the building, density of tags and their spatial distribution. The best cluster with the smallest score is chosen as the target cluster using Equation 8.

$\beta_{G_{r}}^{T_{u}}=\sqrt{\sum_{s=1}^{m}\left(R S S_{S}^{R_{i}}-R S S_{s}^{T_{j}}\right)^{2}}$

$$
\beta=\left[\beta_{R_{i}}^{T_{j}}\right]_{i=1, . . n ; j=1, . . p}
$$




$$
\begin{aligned}
& D_{G_{r}}=\sum_{e=1}^{o_{G_{r}}} \sqrt{\left(x_{R_{e} G_{r}}-x_{G_{r}}^{C}\right)^{2}+\left(y_{R_{e}}^{G_{r}}-y_{G_{r}}^{C}\right)^{2}} \\
& \beta_{G_{r}}^{T_{u}}=\left(\sum_{e=1}^{o_{G_{r}}} \beta_{R_{e}^{G_{r}}}^{T_{u}}\right) / o_{G_{r}} \\
& K_{G_{r}}=w_{D} \times D_{G_{r}}^{\prime}+w_{\beta} \times \beta_{G_{r}}^{T_{u}}
\end{aligned}
$$

$$
\begin{aligned}
& D_{G_{r}}^{\prime}=D_{G_{r}} / \operatorname{Max}\left\{D_{G_{r}}\right\} \\
& \beta_{G_{r}}^{T_{u}}=\beta_{G_{r}}^{T_{u}} / \operatorname{Max}\left\{\beta_{G_{r}}^{T_{u}}\right\} \\
& K_{G_{r}}^{\text {Best }}=\operatorname{Min}\left\{K_{G_{r}}\right\}
\end{aligned}
$$

\section{Localization and Accuracy Calculation}

In our method, weighted averaging of selected reference tags coordinates is used for localization. The weights are calculated based on an empirical function used by Ni et al. (2003). However, the selected reference tags that belong to the best cluster are used for weighted averaging calculations.

Once the target cluster is selected $\left(G_{r}^{\text {Best }}\right)$, the closest reference tag (the one with the most similar signal pattern) within the cluster is chosen $\left(R_{\text {Closest }}^{G_{r}^{\text {Best }}}\right)$. In order to estimate the accuracy of the localization, the chosen reference tag is localized using the same method. Since the coordinates of the reference tags are known, the distance between the estimated location and the actual location can be calculated. The distance shows the error of localization for the closest reference tag. This value approximates the accuracy of localization in the target area.

\section{SIMULATION PROTOTYPE AND CASE STUDY}

A simulation environment is developed in Matlab (MathWorks, 2012) in order to evaluate the proposed methods for various distributions of reference and target tags, data collection points, RSSI behaviours, and the number of readings in each data collection point. Furthermore, new mathematical and procedural techniques (e.g., data filtering, pattern matching techniques, clustering and localization modules) are developed and tested. The Matlab code used in the simulation environment is also used to process real data in the field tests.

The simulator has different modules such as RSSI generator, data filtering, pattern similarity assessment, clustering, localization, and sensitivity analysis. The generation of RSSI values in the simulation uses Monte Carlo approach based on our field test results explained in Motamedi et al. (2011). The simulator simulates an obstacle-free environment where the behaviour of the RFID signals follow the results of our field test in a similar environment.

Figure 3 shows a snapshot of a sample simulation input data with 75 randomly distributed reference and 25 target tags. The small and large stars show the location of reference tags and target tags, respectively. The path that the user with a handheld reader took to localize the target tags is shown by a line. Stars on the path show the data collection points. As shown in the figure, there are eight data collection steps. Figure 3(b) shows the results of one case where target tag 13 is localized with four data collection points. The dark large star is the estimated location of the target based on the clustering method and the white star represents the position of the target calculated by the LANDMARC method. The diameters of the circles around reference tags are inversely proportional to the $\beta$ values. Hence, the bigger the diameter of the circle, the closer the associated reference tag to the target tag. As shown in the figure, the simulation tool is able to identify the closest reference tags to the target and to estimate its location. 


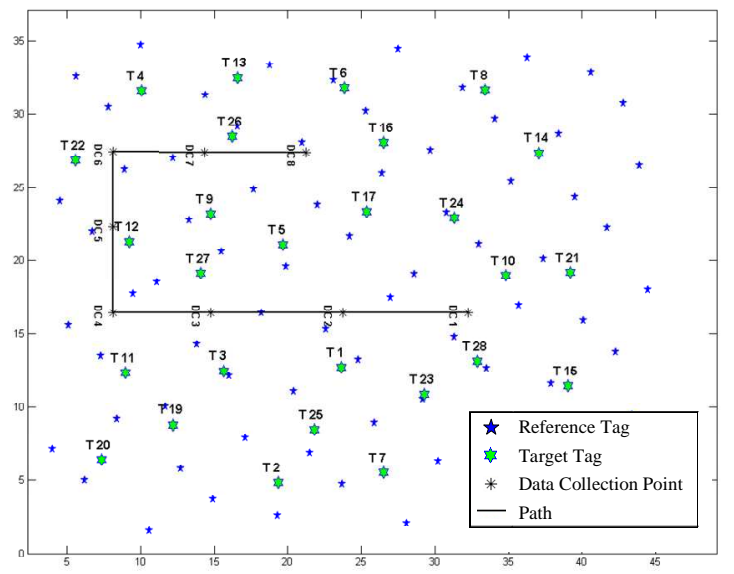

(a) Defining reference and target tags and data collection points

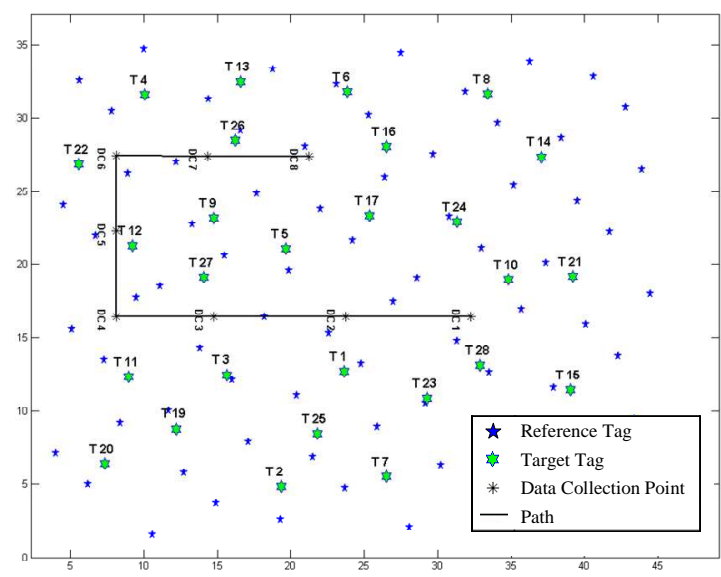

(b) Simulation results for sample target tag (T13)

Figure 3 - Sample simulation input data and results

\section{Localization Accuracy Comparison Using Simulation}

In order to compare the applicability of CMTL method, a comparative study has been performed using our simulation environment. The accuracy of localization using CMTL and LANDMARC methods are compared by developing both approaches in the simulation environment. Clusters of four reference tags and equal weights of one (Equation 7) are used in the simulation environment. Figure 3(a) shows the locations of 25 target tags for one sample simulation setup with 75 reference tags. Three different scenarios for three data collection paths were considered which are a straight path (I-shape) (first four points), an L-shape path (first six points) and a U-shape path (all eight points). For each run of the simulation, a target tag is placed randomly on the map. The simulation is repeated to localize the position of 25 target tags using the above-mentioned data collection paths.

Tables 1 illustrate the average localization error of 25 randomly placed target tags for different data collection paths and reference tag densities based on CMTL and LANDMARC methods. The last row presents the improvement percentage that CMTL provides over the LANDMRC method. For example, in the I-shape data collection scenario, the results show improvements of $18 \%, 17 \%, 34 \%$ and $22 \%$ for the densities of $45,60,75$, and 90 reference tags per area, respectively. The improvement for the cases of U-shape path is small due to the fact that the four closest tags selected in CMTL and LANDMARC methods are mostly identical.

Table 1 - localization accuracy for groups of 4-reference tags and different paths

\begin{tabular}{c|ccc|ccc|ccc|cccc}
\hline $\begin{array}{c}\text { Number of Reference } \\
\text { Tags }\end{array}$ & \multicolumn{4}{c}{$\mathbf{4 5}$} & $\mathbf{6 0}$ & \multicolumn{4}{c}{$\mathbf{7 5}$} & \multicolumn{3}{c}{$\mathbf{9 0}$} \\
\hline $\begin{array}{c}\text { Data Collection Path } \\
\text { Shape }\end{array}$ & $\mathbf{I}$ & $\mathbf{L}$ & $\mathbf{U}$ & $\mathbf{I}$ & $\mathbf{L}$ & $\mathbf{U}$ & $\mathbf{I}$ & $\mathbf{L}$ & $\mathbf{U}$ & $\mathbf{I}$ & $\mathbf{L}$ & $\mathbf{U}$ \\
\hline $\begin{array}{c}\text { LANDMARC Error } \\
(\mathrm{m})\end{array}$ & 7.26 & 2.77 & 1.26 & 7.23 & 2.31 & 1.14 & 7.71 & 1.62 & 1.15 & 7.01 & 1.69 & 1.07 \\
\hline CMTL Error (m) & 5.93 & 1.92 & 1.2 & 6.02 & 1.75 & 1.1 & 5.06 & 1.23 & 1.11 & 5.5 & 1.24 & 1.04 \\
\hline Improvement (\%) & $\mathbf{1 8}$ & $\mathbf{3 1}$ & $\mathbf{5}$ & $\mathbf{1 7}$ & $\mathbf{2 4}$ & $\mathbf{4}$ & $\mathbf{3 4}$ & $\mathbf{2 4}$ & $\mathbf{4}$ & $\mathbf{2 2}$ & $\mathbf{2 7}$ & $\mathbf{2}$ \\
\hline
\end{tabular}




\section{Case Study}

This case study is performed to test the applicability of CMTL method for tracking moveable assets in a multi-tag indoor environment. The Active RFID tags from Identec Solutions (Identec, 2012) with relatively long-range $(100 \mathrm{~m}$ ) are used together with a handheld reader. The test was conducted in an obstacle-free environment where all tags were placed inside one room. The tags were placed on the ground in a grid of $5 \mathrm{~m} \times 7.5 \mathrm{~m}$. A target tag was placed randomly in the room with the distance of $70 \mathrm{~cm}$ from the closest reference tags $\left(R_{9}\right.$ and $R_{12}$ in Figure 4$)$ and data were collected using a handheld reader at six data collection steps forming a U-shaped path for 30 seconds at each data collection step with the frequency of 2 readings per second. Figure 4(b) shows the same setup in the simulation environment. The RSSI values were generated using our signal propagation model (Motamedi et al., 2011) and are compared with the actual measured data. In Figure 4, the diameter of circles around reference tags are inversely proportional to their $\beta$ values. The results show that $R_{12}$ has the least $\beta$ value in both field test and simulation environment. Table 2 shows the comparison between the two localization techniques for the case study. The results of the field test show that localization based on four reference tags using clustering technique is more accurate than the results of the LANDMARC technique. The improvement in accuracy is due to the fact that the LANDMARC's four-nearest reference tags (shown in Figure 4(a)) are different from the ones of the best selected cluster.

Table 2 - Results comparison

\begin{tabular}{crrrrrrrr} 
& \multicolumn{4}{c}{ Field test results } & \multicolumn{3}{c}{ Simulated results } \\
\hline Method & \multicolumn{2}{c}{ CMTL } & \multicolumn{2}{c}{ LANDMARC } & \multicolumn{2}{c}{ CMTL } & \multicolumn{2}{c}{ LANDMARC } \\
\hline Number of Tags & 3 Tags & 4 Tags & 3 Tags & 4 Tags & 3 Tags & 4 Tags & 3 Tags & 4 Tags \\
\hline Error $(\mathrm{m})$ & 0.43 & 0.34 & 0.43 & 0.51 & 0.28 & 0.45 & 0.28 & 0.45 \\
\hline
\end{tabular}

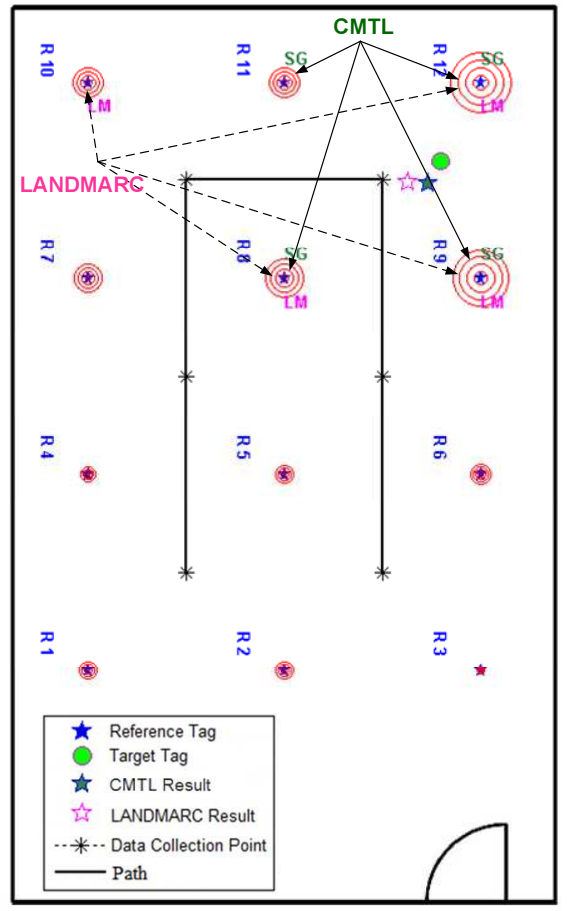

(a) Test results

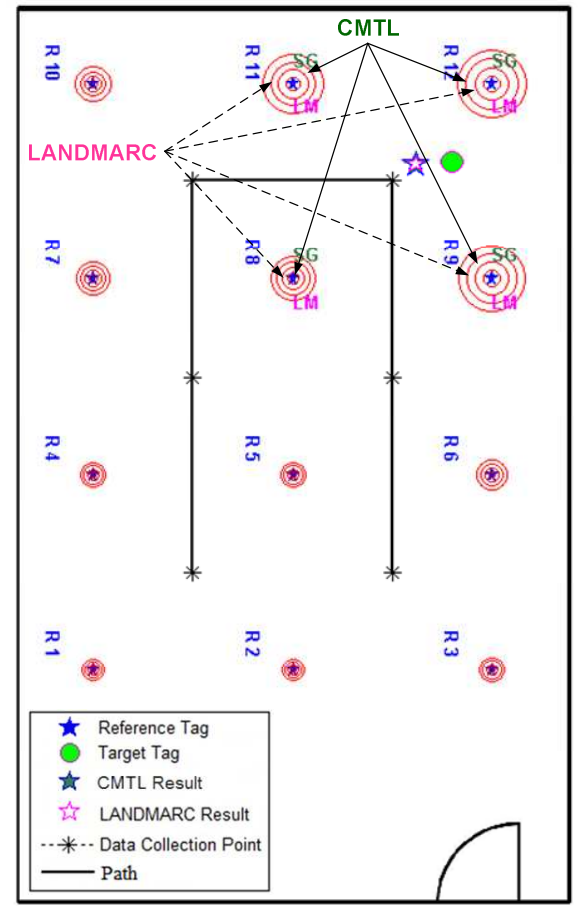

(b) Simulated results

Figure 4 - Comparison of the test and simulation results 


\section{SUMMARY, CONCLUSIONS AND FUTURE WORK}

This paper investigated a method to localize RFID-equipped movable assets in a building using handheld RFID readers. The main advantages of the proposed system are that it can adapt to the changes in the environment, it utilizes available RFID tags in the building, and does not require a fixed RTLS infrastructure for localization. The CMTL method is based on neighbourhood methods. However, CMTL uses a handheld reader as opposed to a fixed number of fixed readers in similar LANDMARC-based methods. Using a handheld reader provides the flexibility to choose the number data collection points and paths. The clustering method introduced major improvements in the accuracy in case of symmetrical settings of reference tags with respect to the data collection path or the cases in which some reference tags randomly show high similarity with the target tag. The results of case studies showed that CMTL is able to estimate the location of the target asset with higher accuracy compared to LANDMARC.

The proposed method can be further improved by applying dynamic segmentation techniques and more advanced signal processing methods for removing noise from logged data. Moreover, other pattern matching and dynamic clustering methods can be employed and compared. Other localization techniques in addition to the weighted averaging can be developed, tested and compared. Furthermore, more in-depth research to evaluate the effects of density and dispersion of reference tags, the number of data collection points, and the number of collected data at each point on the accuracy of the system is required.

\section{REFERENCES}

Aimglobal (2012). What is RFID, Retrieved April 24, 2012, Retrieved from: <http://www.aimglobal.org/technologies/rfid/what_is_rfid.asp>.

Identec (2012). Identec Solutions Inc., from: < http://www.identecsolutions.com/>.

Kanungo, T., Mount, D.M., Netanyahu, N.S., Piatko, C.D., Silverman, R., Wu, A.Y., (2002). An efficient k-means clustering algorithm: analysis and implementation, IEEE Transactions on Pattern Analysis and Machine Intelligence, 24(7), pp. 881-892.

Li, N., and Becerik-Gerber, B. (2011). Performance-based evaluation of RFID-based indoor location sensing solutions for the built environment, Advanced Engineering Informatics, 25(3), pp. 535-546.

MathWorks (2012). Matlab Software, from: < http://www.mathworks.com/products/matlab/>.

Motamedi, A., Hammad, A. (2009). Lifecycle management of facilities components using radio frequency Identification and building information model, ITCon, 14, pp. 238-262.

Motamedi, A., Soltani, M. and Hammad, A. (2012). Localization of RFID-Equipped Assets during the Operation Phase of the Building, ICCCBE2012, Moscow, Russia.

Ni, L. M., Liu, Y. H., Lau, Y. C. and Patil, A.P. (2003). LANDMARC: indoor location sensing using active RFID, 1st IEEE International Conference on Pervasive Computing and Communications, pp. 407-415

Papapostolou, A., and Chaouchi, H. (2011) RFID-assisted indoor localization and the impact of interference on its performance, Network and Computer Applications, 34(3), pp. 902913.

Sanpechuda, T. and Kovavisaruch, L. (2008). A review of RFID localization: Applications and techniques, ECTI-CON, 2, pp. 769,772.

Zhao, Y., Liu, Y., Ni, L.M. (2007). VIRE: active RFID-based localization using virtual reference elimination, 2007 International Conference on Parallel Processing, IEEE, Piscataway, NJ, USA. 